\title{
The NEDD8 conjugation pathway regulates p53 transcriptional activity and head and neck cancer cell sensitivity to ionizing radiation
}

\author{
SÉBASTIEN GUIHARD ${ }^{1,2}$, LUDIVINE RAMOLU ${ }^{1}$, CHRISTINE MACABRE $^{1}$, BOHDAN WASYLYK $^{3}$, \\ GEORGES NOËL ${ }^{1,2}$, JOSEPH ABECASSIS ${ }^{1}$ and ALAIN C. JUNG ${ }^{1}$ \\ ${ }^{1}$ EA3430, Laboratory for Tumor Biology and ${ }^{2}$ Radiation Therapy Department, Paul Strauss Cancer Center, \\ F-67065 Strasbourg Cedex; ${ }^{3}$ IGBMC, UMR 7104 CNRS UDS - U 964 INSERM, F-67404 Illkirch Cedex, France
}

Received February 13, 2012; Accepted April 27, 2012

DOI: 10.3892/ijo.2012.1584

\begin{abstract}
Human papillomavirus (HPV)-related oropharyngeal cancer represents a distinct head and neck squamous cell carcinoma (HNSCC) subpopulation, with improved diseasefree and overall survival. In general, HPV-positive HNSCCs express wild-type TP53, which could explain its increased radiosensitivity. However, the molecular mechanisms underlying this higher sensitivity remain elusive. We have previously shown that HPV-related oropharyngeal carcinomas express decreased levels of the NEDD8-activating enzyme 1/amyloid $\beta$ precursor protein-binding protein 1 (NAEl/APP-BPI) gene. NAE1/APP-BP1 function is required for the NEDDylation of target proteins, and has been shown to be a negative regulator of p53 transcriptional activity. In this study, we addressed the hypothesis that $N A E 1 / A P P-B P 1$ expression levels regulate $\mathrm{p} 53$ activity and cell survival upon ionizing irradiation. We used the radiosensitive and naturally HPV16-infected UPCI:SCC90 cell line and the radioresistant and HPV-negative SQ20B cell line as the control. NAE1/APP-BP1 expression levels were modulated with expression constructs and siRNAs. Radiosensitivity was evaluated with clonogenic survival assays. p53 transcriptional activity was measured with a luciferase assay. The overexpression of NAE1/APP-BPI in UPCI:SCC90 cells resulted in the increased NEDDylation of p53, inhibition of p53 activity and increased cell resistance to ionizing radiation. Conversely, the inhibition of NAEl/APP-BPl expression in SQ20B cells induced p53-dependent cell death after treatment with $\mathrm{X}$-rays. Taken together, these results indicate that NAE1/APP-BPl and NEDDylation are invovled in modulating p53 activity and regulating its role in the response of cells to ionizing radiation. Our findings bring new insights in the molecular mechanisms
\end{abstract}

Correspondence to: Dr Alain C. Jung, Laboratory for Tumor Biology, Paul Strauss Cancer Center, 3 Rue de la Porte de l'Hôpital, F-67065 Strasbourg Cedex, France

E-mail: ajung@strasbourg.unicancer.fr

Key words: human papillomavirus, NEDDylation, p53, head and neck cancer, radiosensitivity underlying the increased radiosensitivity of HPV-related oropharyngeal tumors. This is of importance, as no reliable and robust predictive biomarkers for tumor response to radiotherapy are currently available. These results also have potential clinical significance, as drugs targeting NAE1/APP-BP1 have recently emerged as a novel therapeutic modality in cancer treatment.

\section{Introduction}

The persistent infection of the head and neck epithelium by high-risk human papillomaviruses (HR-HPVs), mainly HPV type 16 , predominantly in the region of the oropharynx, is associated with an increased risk of head and neck squamous cell carcinoma (HNSCC) (1). HR-HPVs define a distinct clinical subpopulation that displays improved disease-free and overall survival (reviewed in ref. 1 and references therein). These observations may be explained by the molecular mechanisms that underlie HPV-driven cell transformation. HR-HPV induces carcinogenesis by interfering with the p53 and pRb signaling pathways (1). The HPV E6 oncoprotein is known to induce p53 ubiquitination and subsequent degradation. The TP53 gene is not mutated in the majority of HPV-related HNSCCs, and data from cell culture assays suggest that non-degraded p53 persisting in HPV-positive cells may be sufficient to induce the expression of p53 target genes and cell death upon treatment with ionizing radiation (2).

Genomic and transcriptomic analyses have shown that HR-HPV-positive HNSCC display specific chromosomal gains and losses, as well as changes in gene expression relative to their HPV-negative counterparts. HR-HPV-related tumors have fewer genomic aberrations, but display specific imbalances, such as a loss of the $16 \mathrm{q}$ region $(3,4)$. Of note, this aberration correlates with improved local control (4). We have previously shown that the expression of the NEDD8-activating enzyme 1/ amyloid $\beta$ precursor protein-binding protein 1 (NAE1/ $A P P-B P 1)$ gene, which is located on chromosome 16q22, is expressed at lower levels in HPV-positive oropharyngeal tumors (3). Together with the UBA3 protein, NAE1/APP-BP1 forms the NEDD8-activating enzyme, which has E1 ubiquitinligase activity, and initiates the NEDD8 conjugation pathway. In this complex, NAE1/APP-BP1 plays the role of a regulatory 
subunit for NEDD8 E1 enzyme activity $(5,6)$. Post-translational NEDD8 conjugation has mainly been described for members of the cullin family of proteins (7). Previously, a function of the NEDD8 conjugation pathway in the regulation of the p53 transcriptional activity has been unraveled: NAE1/APP-BP1 is required for the NEDD8 conjugation of $\mathrm{p} 53$ via MDM2, which results in the inhibition of p53 transcriptional activity without triggering its degradation (8).

In this study, we investigated the possibility that the expression of NAE1/APP-BP1 affects p53-dependent transcriptional activity and sensitivity to ionizing radiation in head and neck cancer cells. We used the naturally HPV16-infected oropharyngeal cell line, UPCI:SCC90, that expresses wild-type p53 (9), and the HPV-negative SQ20B cell line that expresses mutant p53 as the control. UPCI:SCC90 cells are more radiosensitive than SQ20B cells (10). We show that UPCI:SCC90 cells express lower levels of $N A E 1 / A P P-B P 1 \mathrm{mRNA}$, and that the overexpression of $N A E 1 / A P P-B P 1$ in these cells induces radioresistance. Conversely, SQ20B cells become radiosensitive when wild-type TP53 is expressed. The inhibition of NAE1/ $A P P-B P 1$ potentiates the effect of wild-type $\mathrm{p} 53$. These results point to the importance of NEDDylation in the radiosensitivity of tumor cells that express wild-type p53.

\section{Materials and methods}

Cell culture and transfections. The HNSCC SQ20B cell line was purchased from the American Type Culture Collection (ATCC; Rockville, MD, USA). The radioresistant SQ20B cells have been reported to express non-funtional p53 protein (10). The hHNSCC UPCI:SCC90 cell line (9) was a kind gift from Professor Susan Gollin (University of Pittsburgh, Pittsburgh, PA, USA). UPC1:SCC90 cells originate from a HPV16positive oropharyngeal tumor, and bear wild-type TP53 (9). UPCI:SCC90 cells have been shown to be more radiosensitive than SQ20B cells (11). The cells were grown in Dulbecco's modified Eagle's medium (DMEM; PAN Biotech GmbH, Dominique Dutscher, Brumath, France) according to standard conditions.

All transfection experiments were carried out using Lipofectamine $^{\mathrm{TM}} 2000$ (Invitrogen, Fisher Scientific, Illkirch, France) according to the manufacturer's instructions. UPCI:SCC90 cells $\left(5 \times 10^{5}\right)$ were transfected with $4 \mu \mathrm{g}$ of the NAE1/APP-BPl expression vector (12) (kind gift from Dr Dimitris Xirodimas). Mock transfections with $4 \mu \mathrm{g}$ of the empty pcDNA3 vector (Invitrogen) or the empty pCI-neo vector (Promega, Charbonnières-les-bains, France) were used as the negative controls. p53 was overexpressed by transfection of $5 \times 10^{5}$ SQ20B cells with $4 \mu \mathrm{g}$ of pC53-C1N3 plasmid.

siRNA experiments. The NAEl/APP-BPl downregulation experiments were performed using the ON-TARGET plus SMARTpool (L-006401-00-0005; Dharmacon, Fisher Scientific, Illkirch, France). This pool is composed by the combination of 4 single siRNAs: siRNA-1, upgrade siRNA NAE1/APP-BP1 J-006401-05; siRNA-2, upgrade siRNA NAE1/APP-BP1 J-006401-06; siRNA-3, upgrade siRNA NAE1/APP-BP1 J-006401-07; and siRNA-4, upgrade siRNA NAE1/APP-BP1 J-006401-08. The ON-TARGET plus nontargeting pool (D-001810-10-05) was used as the negative control. siRNAs (40 pmol) were transfected into $4 \times 10^{4}$ SQ20B cells using Lipofectamine ${ }^{\mathrm{TM}} 2000$ (Invitrogen) according to the manufacturer's instructions.

RNA extraction and quantitative real-time RT-PCR ( $q R T-P C R)$. RNA was extracted from $5 \times 10^{5}$ cells and cDNA synthesis was performed as previously described (3). Cycle threshold $(\mathrm{Ct})$ levels were normalized to the average $\mathrm{Ct}$ values of 3 internal controls (housekeeping genes): ubiquitin B (UBB), 18S rRNA and hypoxanthine phospho-ribosyltransferase 1 (HPRT1). Primers specific for the genes of interest were used: $H R-H P V 16$ E6/E7 mRNA forward, 5'-GTTACTGCGACGTGAGGTA TATG-3' and reverse, 5'-CATTTATCACATACAGCATATGG ATTC-3' (13); NAE1/APP-BPl forward, 5'-CTGGAAAGCT GCTCAAGG-3' and reverse, 5'-TCTTCTCCGCTGACCT GATT-3'; $C D K N 2 A^{p 16}$ forward, 5'-GCTGCCCAACGCACCGA ATA-3' and reverse, 5'-ACCACCAGCGTGTCCAGGAA-3'; WAF $1 / C I P 1^{\mathrm{p} 21}$ forward, 5'-ATGAAATTCACCCCCTTTCC-3' and reverse, 5'-CCCTAGCTGTGCTCACTTC-3'; PUMA forward, 5'-ACGACCTCAACGCACAGTACGA-3' (14) and reverse, 5'-GTAAGGGCAGGAGTCCCATGATGA-3' (14); MDM2 forward, 5'-GGTGGGAGTGATCAAAAGGA-3' and reverse, 5'-GTGGCGTTTTCTTTGTCGTT-3'; HPRT1 forward, 5'-TGCTCGAGATGTGATGAAGG-3' and reverse, 5'-GT CCCCTGTTGACTGGTCATT-3'; UBB forward, 5'-GCTTTG TTGGGTGAGCTTGT-3' and reverse, 5'-CGAAGATCTGCA TTTTGACCT-3'; $18 S$ rRNA forward, 5'-TGTGGTGTTGA GGAAAGCAG-3' and reverse, 5'-TCCAGACCATTGGCT AGGAC-3'. Gene expression levels observed in the nonirradiated mock-transfected cells were artificially set to 100 , and other expression values were normalized with respect to this level. Differences were considered statistically significant when $\mathrm{p}<0.05$ (ANOVA).

Irradiation procedures, clonogenic survival assay and surviving fraction at 2 Gy (SF2) calculation. Cells in the exponential growth phase were detached, counted and plated in 6-well plates in $2 \mathrm{ml}$ of growth medium 24 to $48 \mathrm{~h}$ prior to irradiation, in order to allow them to attach and undergo transfection. X-ray irradiation was performed with $6 \mathrm{MV}$ photons at the Department for Radiation Therapy of the Paul Strauss Cancer Centre (Strasbourg, France). Clonogenic assays were performed as previously described (15). In brief, cells were collected $24 \mathrm{~h}$ after irradiation, diluted, seeded and allowed to grow for up to 3 to 4 weeks after irradiation. Clones were stained with $0.05 \%$ crystal violet (Sigma-Aldrich, Lyon, France) in a 5\% ethanol solution, and positive colonies (>64 cells) were counted. The SF2 was calculated by dividing the number of positive colonies by the number of cells that were seeded, multiplied by the plating efficiency. The plating efficiency was calculated by dividing the number of positive colonies that grew in the absence of irradiation by the number of cells that were seeded. Differences were considered statistically significant when $\mathrm{p}<0.05$ (ANOVA).

Western blot analyses. Cells $\left(4 \times 10^{4}\right.$ to $\left.5 \times 10^{6}\right)$ were harvested in lysis buffer (150 mM NaCl, 1\% NP-40, $50 \mathrm{mM}$ Tris-Cl pH 8.0, $0.5 \%$ DOC, $0.1 \%$ SDS) and proteins were analyzed by SDS-PAGE according to standard methods. Proteins were detected with the following antibody dilutions: polyclonal mouse AO1 anti- 
APP-BP1 (1:1000; Abnova, Tebu-Bio, Le-Perray-en-Yvelines, France), monoclonal mouse DO1 anti-p53 (1:1000; Santa Cruz Biotechnologies, CliniSciences, Montrouge, France), polyclonal rabbit anti-p53 FL-393 (1:200; Santa Cruz Biotechnologies), anti-NEDD8 rabbit polyclonal (1:2000; Alexis Biochemicals, Enzo Life Sciences, Villeurbanne, France), mouse monoclonal CM1 anti- $\beta$ actin (1:1000; Abnova), anti- $\beta$ tubulin (1:5000; Sigma-Aldrich), enhanced chemiluminescence (ECL) sheep anti-mouse IgG, HRP-conjugated (1:10000; GE Healthcare, Saclay, France), and anti-rabbit IgG, HRP-linked antibodies (1:20000; Cell Signaling, Ozyme, Saint-Quentin-en-Yveline, France). Proteins were revealed with the ECL western blot analysis system (GE Healthcare) according to the manufacturer's instructions. Quantification of the signals was performed using Image J software (National Institutes of Health, Bethesda, MD, USA) (16,17).

p53 transcriptional activity assay. With the use of the Lipofectamine $^{\mathrm{TM}} 1000$ transfection system, 5x10 ${ }^{5}$ UPCI:SCC90 cells were co-transfected with $0.75 \mu \mathrm{g}$ of PG13-Luc plasmid (kind gift from Dr Arnold Levine) or MG15-Luc plasmid, $0.75 \mu \mathrm{g}$ of RSV-LacZ vector [used as a transfection efficiency control (18)], and increasing amounts $(0,1.0,1.5,2.5 \mu \mathrm{g})$ of either the NAE1/APP-BP1 expression vector or of the empty vector (pcDNA3). The total amount of transfected plasmid was made up to $4 \mu \mathrm{g}$ with pUC19 (New England Biolabs). Cells were harvested $36 \mathrm{~h}$ after transfection in lysis buffer $(25 \mathrm{mM}$ Tris-phosphate, $\mathrm{pH}$ 7.8; 2 mM EDTA; 1 mM DTT; $10 \%$ glycerol; $1 \%$ Triton $\mathrm{X}-100$ ), and luciferase activity was evaluated by mixing $50 \mu \mathrm{l}$ of protein extract with luciferase assay buffer (265 $\mu \mathrm{M}$ ATP; $235 \mu \mathrm{M}$ luciferin; $135 \mu \mathrm{M}$ coenzyme A; $20 \mathrm{mM}$ tricine; $1.07 \mathrm{mM} \mathrm{MgCl}$; $2.70 \mathrm{mM} \mathrm{MgSO}_{4} ; 0.10 \mathrm{mM}$ EDTA; $33.3 \mathrm{mM}$ DTT) using a MikroWin 2000 microplate reader (Mikrotek Laboratories GmbH, Overath, Germany). The luminescence levels were normalized to the $\beta$-galactosidase activity from the RSV-LacZ control plasmid that was measured with an $o$-nitrophenyl- $\beta$-galactoside (ONPG) colorimetric reaction. In brief, $\beta$-galatcosidase activity was evaluated by mixing $25 \mu \mathrm{l}$ of cell extract with $150 \mu \mathrm{l}$ of ONPG staining solution $(0.666 \mathrm{mg} / \mathrm{ml}$ ONPG; $2 \% \beta$-mercaptoethanol; $60 \mathrm{mM} \mathrm{Na}{ }_{2} \mathrm{HPO}_{4} ; 40 \mathrm{mM}$ $\mathrm{NaH}_{2} \mathrm{PO}_{4} ; 10 \mathrm{mM} \mathrm{KCl} ; 1 \mathrm{mM} \mathrm{MgSO}{ }_{4}$ ), and $o$-nitrophenol was monitored on a Berthold 900 spectrophotometer using Gen5 Data analysis software. Fluorescence observed in cells transfected with $0 \mu \mathrm{g}$ of $N A E 1 / A P P-B P 1$ expression vector was set at a value of 100 , and other measures were normalized with respect to that point. Differences were considered significant when $\mathrm{p}<0.05$ (Student-Newman-Keuls test for pairwise comparisons).

Immunoprecipitations. Cells $\left(1.5 \times 10^{6}\right)$ were harvested in denaturing lysis buffer (1\% SDS, 5 mM EDTA, $10 \mathrm{mM} \mathrm{DTT,} 15 \mathrm{U} / \mathrm{ml}$ DNase, PMSF). A total of $5 \mu \mathrm{l}$ of each cell lysate was diluted in $45 \mu \mathrm{l}$ of non-denaturing buffer $(20 \mathrm{mM}$ Tris- $\mathrm{HCl} \mathrm{pH} 8.0$, $137 \mathrm{mM} \mathrm{NaCl}, 10 \%$ glycerol, 1\% Triton X-100, 2 mM EDTA, PMSF). Diluted cells lysates were pre-incubated with $0.4 \mu \mathrm{g}$ of anti-p53 antibody (DO1 anti-p53; Santa Cruz Biotechnologies) or with $2 \mu \mathrm{l}$ of anti-NEDD8 antibody (Alexis Biochemicals). A total of $10 \mu \mathrm{l}$ of Protein A/G PLUS-Agarose Immunoprecipitation Reagent (sc-2003; Santa Cruz Biotechnologies) was further mixed with extracted protein, pelleted and resuspended in loading buffer and subjected to western blot analysis with an
anti-NEDD8 antibody and a polyclonal rabbit anti-p53 FL-393 antibody (Santa Cruz Biotechnologies). Normal mouse IgG-AC (10 $\mu$ l) (sc-2343; Santa Cruz Biotechnologies) was incubated with protein lysate as the negative control.

Apoptosis detection by Annexin V staining. UPCI:SCC90 cells were transfected and irradiated as described above. Annexin V staining was monitored by flow cytometry on an Agilent Bioanalyzer 2100 (Agilent Technologies Deutschland, Waldbronn, Germany), according to the manufacturer's instructions. In brief, cells were incubated first with Annexin V-Biotin (Annexin V-Biotin apoptosis detection kit, Oncogene Research Product, Merck Chemicals, Nottingham, UK), and later with streptavidin-Cy5 (Amersham, GE Healthcare). In order to discriminate early apoptotic cells from dead cells, samples were counterstained with Calcein-AM (Molecular Probes, Invitrogen, Cergy Pontoise, France). Cell samples were loaded on Cell Fluorescence LabChip ${ }^{\circledR}$ kits, and flow cytrometry data were analyzed with Agilent Cell Fluorescence software. Data were analyzed with one-way ANOVA, together with a StudentNewman-Keuls test of all pairwise possible comparisons, and differences were considered significant when $\mathrm{p}<0.05$.

\section{Results}

NAE1/APP-BP1 gene and protein expression are lower in the UPCI:SCC90 cells than in SQ20B cells. In order to evaluate the involvement of $N A E 1 / A P P-B P 1$ gene expression levels in HR-HPV-positive oropharyngeal squamous cell carcinoma radiosensitivity, we selected 2 different cell lines: the HPV-negative SQ20B cell line that originates from a laryngeal tumor, harboring a TP53 mutant allele and that is radioresistant (10); and the HR-HPV16-positive UPCI:SCC90 cell line that was established from an oropharyngeal squamous cell carcinoma, that bears a wild-type TP53 locus and is radiosensitive as compared to the SQ20B cell line (11). Using a qRT-PCR approach with 3 independent total RNA extracts from the 2 cell lines, we confirmed that the mRNA that encodes the HPV16 E6 and E7 oncoproteins is expressed in UPCI:SCC90 cells, whereas it was not detectable in RNA extracts from SQ20B cells (Fig. 1A). As expected, the expression of the CDKN $2 A^{p 16}$ gene, which is used as a surrogate marker of HPV infection, was found to be dramatically increased in the UPCI:SCC90 cells as compared to the SQ20B cells (Fig. 1A). These results confirm that HPV16 is biologically active in UPCI:SCC90 cells. We measured the expression of WAF1/CIP1 $1^{p 2 l}$, whose expression is regulated by $\mathrm{p} 53$. In agreement with their respective TP53 statuses, the expression of WAFI/CIP1 ${ }^{p 21}$ was found to be higher in the UPCI:SCC90 cells as compared to the SQ20B cells (Fig. 1A). Finally, the expression of the NAE1/ $A P P-B P 1$ gene was found to be lower in the UPCI:SCC90 cells than in the SQ20B cells. We further evaluated the expression levels of the NEA1/APP-BP1 protein in these 2 cells lines by western blot analysis. Blots were probed with an anti-NEA1/ APP-BP1 antibody, and quantification of the signals obtained from total protein extracts confirmed that the NEA1/APP-BP1 protein displays higher expression levels in SQ20B cells than in UPCI:SSC90 cells (Fig. 1B). Thus, these 2 cell lines display NAE1/APP-BP1 expression patterns that are similar to those reported in HR-HPV-positive and HPV-negative HNSCC. They 
A

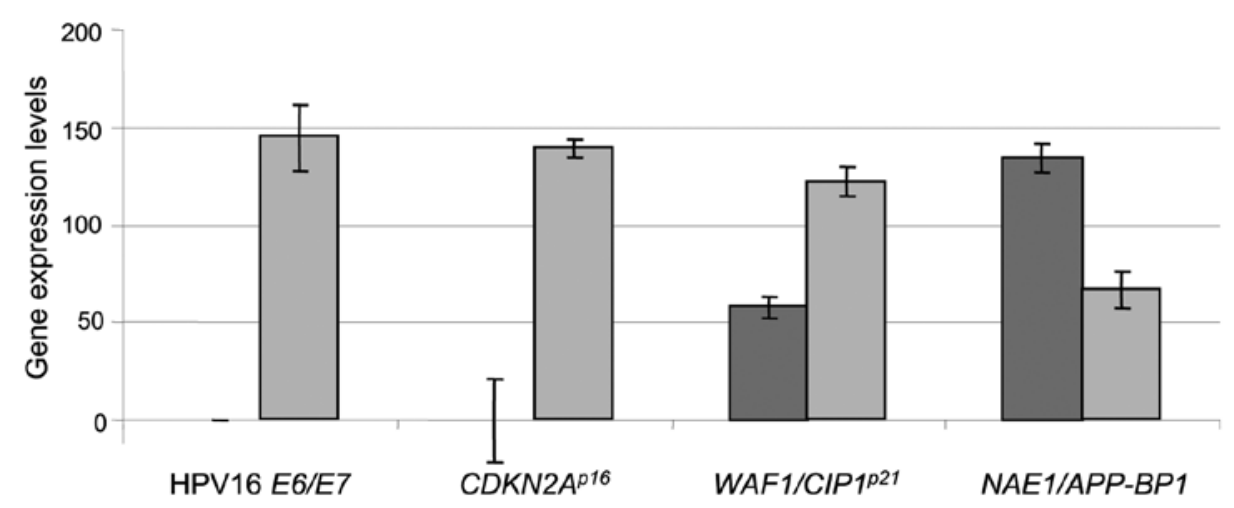

SQ20B UPCI:SCC90

B

a

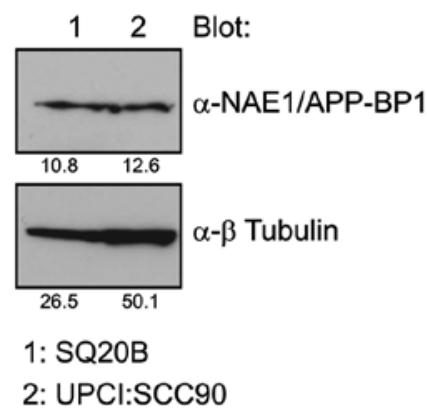

b

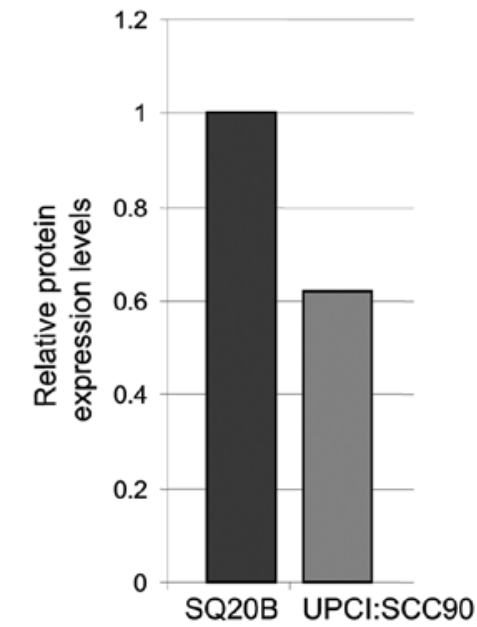

Figure 1. qRT-PCR analysis of gene expression levels in the UPCI:SCC90 and SQ20B cell lines. (A) The expression levels of HPV16 E6/E7, CDKN2A $A^{p 16}$, $W A F 1 / C I P P^{p 21}$ and NAE1/APP-BP1 mRNA were measured in UPCI:SCC90 and SQ20B cell extracts using specific primers and a SYBR-Green-based qRTPCR approach. Mean expression levels and standard deviations were calculated with the data from 3 independent experiments. Results are presented in arbitrary units. (B-a) NEA1/APP-BP1 protein levels were evaluated by western blot analyses of UPCI:SCC90 and SQ20B cell extracts (upper panel). Protein loads were evaluated by probing the membrane with an anti- $\beta$ tubulin antibody (lower panel). Numbers under the gel refer to the quantification of the signal. (B-b) Protein expression levels in SQ20B and UPCI:SCC90 cells as calculated with the normalization to the loading control. Value observed in SQ20B cells has been set to 1 .

were used to evaluate the influence of the $N A E 1 / A P P-B P 1$ gene on their response to ionizing radiation.

Forced expression of NAE1/APP-BPI enhances resistance to ionizing radiation. We examined the effect of modulating NAE1/APP-BP1 expression levels in UPCI:SCC90 cells on clonogenic survival. NAE1/APP-BPI was overexpressed by the transfection of an expression vector. Transfection with the $N A E 1 / A P P-B P 1$ construct resulted in an increase in NAE1/ $A P P-B P 1$ mRNA levels compared to the mock-transfected cells, as measured by qRT-PCR in 3 independent extracts of cells harvested $24 \mathrm{~h}$ after transfection (Fig. 2A). A western blot analysis of protein extracts from the same cells, using an anti-NAE1/APP-BP1 antibody, showed that NAE1/APP-BP1 protein levels also increased (Fig. 2B). In a clonogenic survival assay, the cells were irradiated with a single 2-Gy dose of $\mathrm{X}$-rays, and the number of cells that were able to grow colonies was measured. Non-irradiated cells were used as the control to calculate the fraction of cells that survived the treatment. The SF2 of mock-transfected cells and that of cells that overexpressed NAE1/APP-BP1, calculated from 6 independent measures, was 0.36 and 0.89 , respectively (Table I; $\mathrm{p}<0.001$ ), showing that NAEl/APP-BPl overexpression augments resistance to ionizing radiation in UPCI:SCC90 cells.

Downregulation of NAE1/APP-BP1 increases radiosentivity in a p53-dependent manner. We reasoned that if NAE1/APP-BPI is involved in cell survival after irradiation, then the response of SQ20B cells to ionizing radiation may be modulated by both restoring functional TP53 and downregulating NAE1/APP-BPI expression. Consequently, SQ20B cells were co-transfected with a wild-type TP53 expression vector and siRNAs that specifically target $N A E 1 / A P P-B P 1$. A non-related siRNA was used as the negative control. SQ20B cells bear a point mutation in the TP53 gene that results in the production of a non-functional p53 protein, which can be detected by western 
A

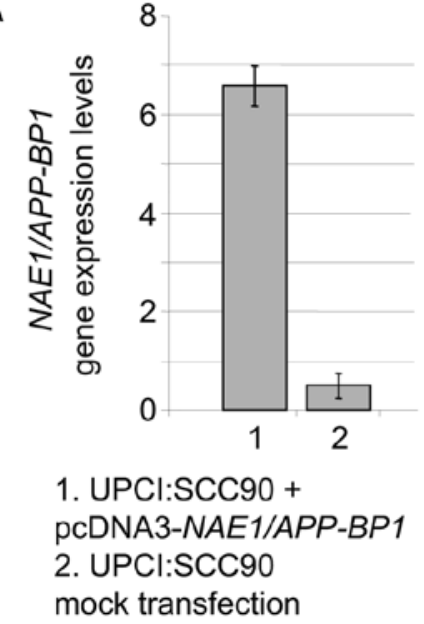

B
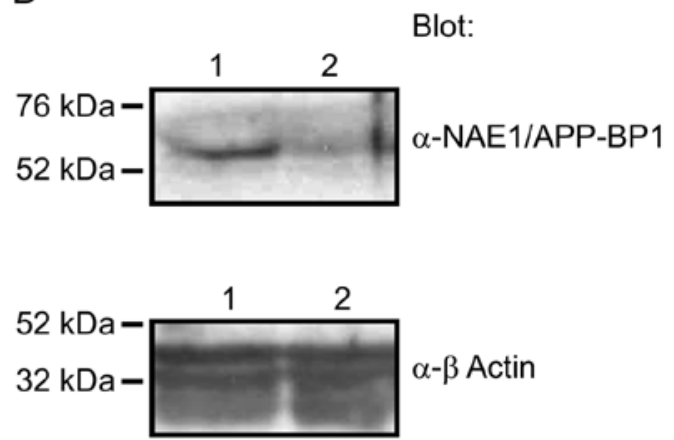

1. UPCI:SCC90 + pCDNA3-NAE1/APP-BP1

2. UPCI:SCC90 mock transfection

Figure 2. Overexpression of NAE1/APP-BP1 in UPCI:SCC90 cells. (A) UPCI:SCC90 cells were transfected with a NAE1/APP-BP1 expression construct or with an empty vector as the negative control. The expression levels of the NAE1/APP-BP1 gene were measured by qRT-PCR in 3 independent RNA extracts from transfected UPCI:SCC90 cells. Mean expression levels and standard deviations were calculated with the data from 3 independent experiments. Results are presented in arbitrary units. (B) Western blot analysis of the expression of the NAE1/APP-BP1 protein (60 kDa) in extracts of UPCI:SCC90 cells that were transfected with either the NAE1/APP-BP1 expression construct (lane 1; upper panel) or with the empty vector as a negative control (lane 2; upper panel). Protein loads were evaluated by probing the membrane with an anti- $\beta$ actin antibody (lanes 1 and 2 ; lower panel).

Table I. Effects of NAE1/APP-BP1 and TP53 expression on the fraction of UPCI:SCC90 and SQ20B cells that survived after irradiation with 2 Gy X-Rays (SF2).

Cell transfection

SF2 Gy

p-value

UPCI:SCC90 cells

Mock transfection

$0.36 \pm 0.16$

$<0.001$

pcDN3-NAE1/APP-BPl

$0.89 \pm 0.07$

SQ20B cells

Single transfection

$\begin{array}{ll}\text { Mock transfection } & 0.46 \pm 0.01\end{array}$

$\begin{array}{ll}\text { Control siRNA } & 0.64 \pm 0.10\end{array}$

NS

$N A E 1 / A P P-B P 1$ siRNA

$0.53 \pm 0.12$

NS

pC53-C $1 \mathrm{~N}_{3}$

$0.35 \pm 0.02$

0.030

Double transfection
pC53-C1N $3+$ control siRNA
$0.46 \pm 0.14$
pC53-C1N ${ }_{3}+N A E 1 / A P P-B P 1$ siRNA
$0.26 \pm 0.04$

0.045

UPCI:SCC90 cells were transfected with the NAE1/APP-BP1 expression construct or with the empty vector as the negative control (mock transfection). SQ20B cells, used as a radioresistant control cell line, were single-transfected with the following constructs: empty vector (mock transfection), NAE1/APP-BP1 unrelated control siRNA, anti-NAE-1/APP-BP1 siRNA, wild-type p53 expression construct. SQ20B cells were doubly-transfected with the wild type p53 expression construct and either the NAE1/APP-BP1 unrelated control siRNA or the anti-NAE-1/ $A P P-B P 1$ siRNA. The cells were irradiated with $2 \mathrm{~Gy}$ of $\mathrm{X}$-Rays, and SF2s and standard deviations were calculated with the data from 3 independent experiments, with 6 independent measurements per experiment. Differences were considered as significant when $p<0.05$. NS, not significant.

blot analysis (Fig. 3A, lanes 1 and 3). The transfection of SQ20B cells with an expression vector for wild-type TP53 further increased p53 levels. Non-irradiated and irradiated transfected cells displayed increased amounts of p53 as compared to the mock-transfected cells (Fig. 3A, lanes 2 and 4). The SF2 of mock-transfected SQ20B cells and that of SQ20B cells that overexpressed p53, was 0.46 and 0.35 , respectively (Table I; $\mathrm{p}=0.030$ ), indicating that exogenous $\mathrm{p} 53$ function is functional in SQ20B cells. The expression of the NAE1/APP-BP1 protein was downregulated up to 48 and $72 \mathrm{~h}$ after transfecting SQ20B cells with an anti-NAE1/APP-BP1 siRNA pool (Fig. 3B, lane 7). This pool is composed of 4 individual siRNAs, which 
A

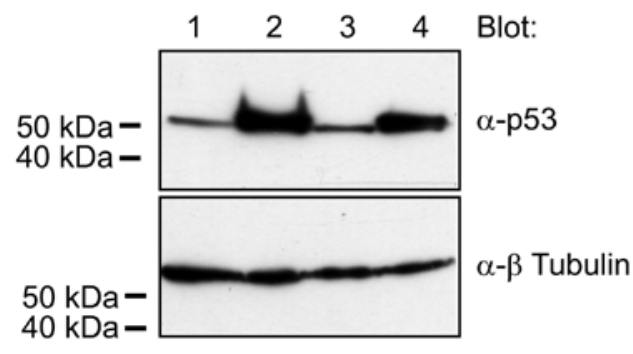

1. SQ20B mock transfection; 0 Gy

2. SQ20B + p53; 0 Gy

3. SQ20B mock transfection; 2 Gy

4. SQ20B + p53; 2 Gy

\section{B}

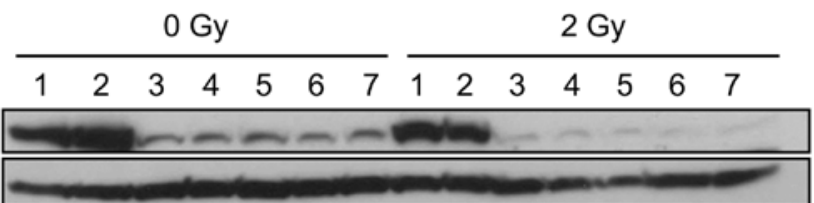

$48 \mathrm{~h}$ after transfection

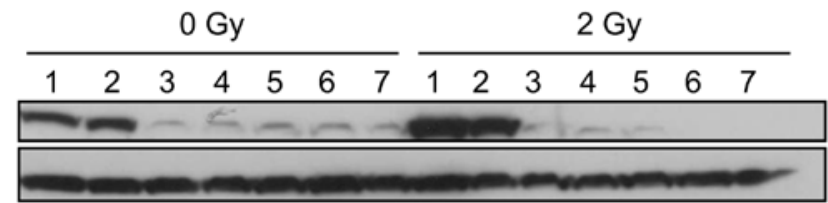

$72 \mathrm{~h}$ after transfection
Blot:

$\alpha-N A E 1 / A P P-B P 1$ $\alpha-\beta$ Tubulin $\alpha-N A E 1 / A P P-B P 1$ $\alpha-\beta$ Tubulin

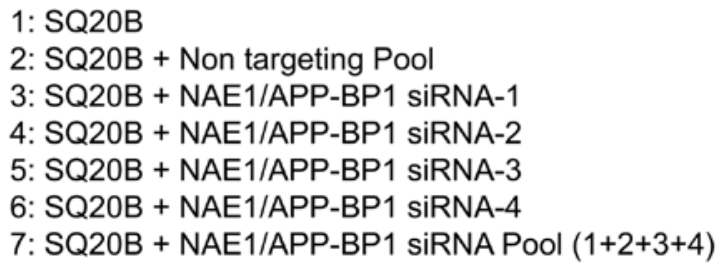

Figure 3. Western blot analysis of p53 and NAE1/APP-BP1 protein expression in SQ20B cells. (A) SQ20B cells were transfected with the p53 expression construct (lanes 2 and 4) or with the empty vector as the negative control (lanes 1 and 3). Cells were either non-irradiated (lanes 1 and 2) or irradiated with $2 \mathrm{~Gy}$ $\mathrm{X}$-rays (lanes 3 and 4). p53 protein (53 kDa) levels were evaluated by western blot analysis of SQ20B cells extracts (upper panel). The membranes were probed with an anti- $\beta$ tubulin antibody to control for loading (lower panel). (B) SQ20B cells were not transfected (negative control, lane 1), or transfected with an anti-NAE1/APP-BP1 siRNA pool (lane 7), with the deconvoluted siRNA pool (lane 3, anti-NAE1/APP-BP1 siRNA-1; lane 4, siRNA-2; lane 5, siRNA-3; lane 6, siRNA-4; or lane 2, with an unrelated control siRNA. Cells were irradiated with a 2 Gy X-ray dose, or non-irradiated as the control. Cells were harvested 48 and $76 \mathrm{~h}$ after transfection. NAE1/APP-BP1 protein levels were detected by western blot analysis of SQ20B cells extracts (upper panel). Loading was controlled by probing the membrane with an anti- $\beta$ actin antibody (lower panel).

all were found to efficiently downregulate the expression of the NAE1/APP-BP1 gene (Fig. 3A, lanes 3-6). Transfection with a non-related siRNA, used as the negative control, had no effect on NAE1/APP-BP1 protein levels (Fig. 3B, lane 2). Neither the anti-NAEl/APP-BP1 siRNA, nor the control siRNA increased cell sensitivity to ionizing radiation. The SF2 in these conditions was 0.53 and 0.64 , respectively (Table I). Strikingly, the irradiation of SQ20B cells that were co-transfected with wildtype TP53 and anti-NAE1/APP-BPI siRNA resulted in a drop in the SF2 to 0.26 , whereas cells that were co-transfected with TP53 and the control siRNA displayed a SF2 of 0.46 (Table I; $\mathrm{p}=0.045$ ). Taken together, these observations suggest that the inhibition of $N A E 1 / A P P-B P 1$ in SQ20B cells restores cell sensitivity to ionizing radiation in a 553 -dependent manner.

Overexpression of NAE1/APP-BP1 in UPCI:SCC90 cells inhibits 53 transcriptional activity and impairs apoptosis. In order to examine whether the modulation of $N A E 1 / A P P-B P 1$ expression levels in UPCI:SCC 90 cells has an effect on $\mathrm{p} 53$ transcriptional activity, we used the pG13-Luc reporter, in which the gene that encodes the luciferase protein is under the control of p53-dependent cis-regulatory elements. UPCI:SCC90 cells were co-transfected with equal amounts of pG13-Luc and increasing amounts of either the NAEl/APP-BPI expression vector, or the empty control vector. Cells were harvested $36 \mathrm{~h}$ after transfection and luciferase activity was measured with 3 independent protein extracts. There was no change in p53 transcriptional activity in the cells that were mock-transfected with various amounts of control vector (Fig. 4A, left histograms). By contrast, p53 transcriptional activity was markedly and significantly repressed in UPCI:SCC90 cells transfected with increasing amounts of the NAE1/APP-BPI vector, in a dose-dependent manner (Fig. 4A; 1.5 and $2.5 \mu \mathrm{g}$ of $N A E 1 /$ $A P P-B P 1$ vector; $\mathrm{p}<0.05)$. In the control experiment, we used 
the MG15-Luc reporter plasmid, which contains mutated p53-binding elements upstream of the luciferase gene. The fluorescence that we observed was markedly reduced, and no differences were observed between the cells transfected with either the empty plasmid or the NAE1/APP-BP1 expression construct (Fig. 4A, right histograms). The UPCI:SCC90 mock-transfected cells displayed rapid and significant $(\mathrm{p}<0.05)$ induction of $\mathrm{p} 53$ target genes, such as $W A F 1 / C I P l^{p 21}$, PUMA and MDM2, 30 min after a 2-Gy irradiation (Fig. 4B). This induction was not observed in UPCI:SCC90 cells that overexpressed NAEI-APP-BPI (Fig. 4B). In order to evaluate whether this modulation of the p53 dependent transcriptional activity depends on a NAE1/APP-BP1-dependent increase in NEDDylation, we performed a western blot analysis to detect NEDDylated p53 in proteins extracted under denaturating conditions. NEDD8-conjugated proteins were immunoprecipitated with a 53 antibody, and the blots were probed with an anti-NEDD8 antibody (Fig. 4C-a). Conversely, NEDD8 was immunoprecipitated from the protein extracts, and the blots were then probed with an anti-p53 antibody (Fig. 4C-b). NEDDylated proteins were present in similar amounts in the extracts from the mock-transfected cells and from the cells transfected with NAE1/APP-BP1 (Fig. 4C-c). In both cases, we observed a band whose size corresponded to NEDDylated p53 (approximately $63 \mathrm{kDa}$; Fig. 4C-a and -b; asterisk). This signal was stronger in the UPCI:SCC90 cells that overexpressed NAE1/APP-BP1 than in the mock-transfected UPCI:SCC90 cells (Fig. 4C-a and -b). No detectable signal corresponding to a NEDDylated p53 protein was observed when transfected UPCI:SCC90 cell lysates were immunoprecipitated with an unrelated IgG (Fig. 4C-a). These results indicate that the overexpression of NAE1/APP-BPI in UPCI:SCC90 cells results in an increased NEDDylation of p53. These observations are consistent with the decreased transcriptional activity of p53 (Fig. 4A and B).

We evaluated whether the modulation of radiosensitivity by $N A E 1 / A P P-B P 1$ expression levels and p53 activity is associated with changes in apoptosis in the control and transfected UPCI:SCC90 cells, prior to and $6 \mathrm{~h}$ following a 4-Gy irradiation. A flow cytometry approach was used in which living and dead cells were discriminated by staining with a calcein probe, and apoptotic cells by Annexin V. Calcein-positive and -negative cells were cross-gated to cells that displayed high levels of Annexin $\mathrm{V}\left(>10^{2} ;\right.$ Fig. $\left.5 \mathrm{~A}\right)$, and the proportion of each cell category was counted. The graph represents the statistical analysis of 3 independent experiments (Fig. 5B). The proportion of calcein-negative Annexin V-positive cells was found to be decreased when $N A E 1 / A P P-B P 1$ was overexpressed in irradiated UPCI:SCC 90 cells as compared to irradiated mocktransfected cells $(\mathrm{p}<0.05)$. Taken together, these observations suggest that NAE1/APP-BPI regulates cell sensitivity to ionizing radiation by modulating apoptosis.

\section{Discussion}

A hallmark of patients bearing HR-HPV-positive HNSCCs is improved disease-free and overall survival as compared to their HPV-negative counterparts (1), which may be due to increased tumor sensitivity to chemo- and radiation-therapy (19). We have previously described the HPV-positive tumor- specific loss of genetic material in the 16q region (3), which has been reported to be linked to improved local control (4). We have shown that the gene that encodes NAE1/APP-BP1, and which is located on chromosome 16q22, is expressed at lower levels in HPV-positive lesions (3). In the present study, we addressed the role of NAEl/APP-BPI in the regulation of the cellular response to ionizing radiation in HPV-positive and -negative cell lines. NAE1/APP-BP1 is a negative regulator of p53 transcriptional activity. The majority of HR-HPV-related oropharyngeal carcinomas express wild-type TP53 (1) and have a decreased expression of NAEl/APP-BPI (3). Thus, we reasoned that the loss of NAE1/APP-BP1 may be responsible for the increased p53 activity and subsequent tumor cell death upon genotoxic stress.

Similar to the data that has previously been presented for HPV-positive oropharyngeal tumors, in the present study, we show that the expression of NAEl/APP-BPI is downregulated in radiosensitive UPCI:SCC90 cells (11) as compared to SQ20B radioresistant cells (10). The radiosensitive phenotype of UPCI:SCC90 cells was rescued by the overexpression of $N A E 1 / A P P-B P 1$, and conversely, the radioresistance of SQ20B cells was inhibited by the decreasing NAEl/APP-BPI expression. This effect appears to be p53-dependent.

The NEDDylation of p53 in other cancer cell lines has been reported. FBXO11, a component of the SCF E3 ubiquitin-ligase complex, has NEDD8-ligase activity. It physically interacts with $\mathrm{p} 53$ in the human lung carcinoma H1299 cell line, triggers p53 NEDDylation and inhibits its transcriptional activity (20). $N A E 1 / A P P-B P 1$ expression levels correlate with cell death in Drosophila melanogaster. Clones of homozygous $d A P P-B P I$ mutant cells, generated in heterozygous surrounding wild-type tissue, have an unusual small size due to a reduction in the number of cells. This phenotype is due to a dramatic increase of apoptosis in mutant cells (21).

The NEDD8 conjugation pathway has been implicated in the regulation of the activity of the SCF ubiquitin-ligase complex $(7,22)$. Of note, a link between the activity of the SCF complex and sensitivity to ionizing radiation has recently been established (23). The sensitive to apoptosis gene (SAG) protein is a component of the SCF E3 ubiquitine ligase that is overexpressed in certain human cancers. The silencing of SAG in cancer cell lines impairs cell growth and colony formation, and results in increased sensitivity to radiation. Of note, the pro-apoptotic protein, NOXA, has been found to be more abundant in cells in which $S A G$ expression is downregulated, and apoptosis is increased, suggesting that SAG is required for the degradation of NOXA. In our study, we show that the NEDD8 conjugation pathway has an influence on p53-dependent radiation-induced apoptosis. However, we cannot rule out the possibility that the effects observed are also partially linked to effects of the SCF complex on the turn-over of anti- or pro-antiapoptotic factors and cell cycle regulators (22). In addition, it has previously been shown that TAp73 transcriptional activity is inhibited by NEDDylation in the ts 41 or wild-type $\mathrm{CHO}$ cells (24). Therefore, we cannot exclude that the inhibition of TAp73 in transfected UPCI:SCC90 cells partially accounts for the effect of NAE1/APP-BP1 on luciferase and p53 target gene expression.

The radiosensitivity of cancer cells varies during the cell cycle $(25,26)$. However, we did not observe changes in the G1 
A

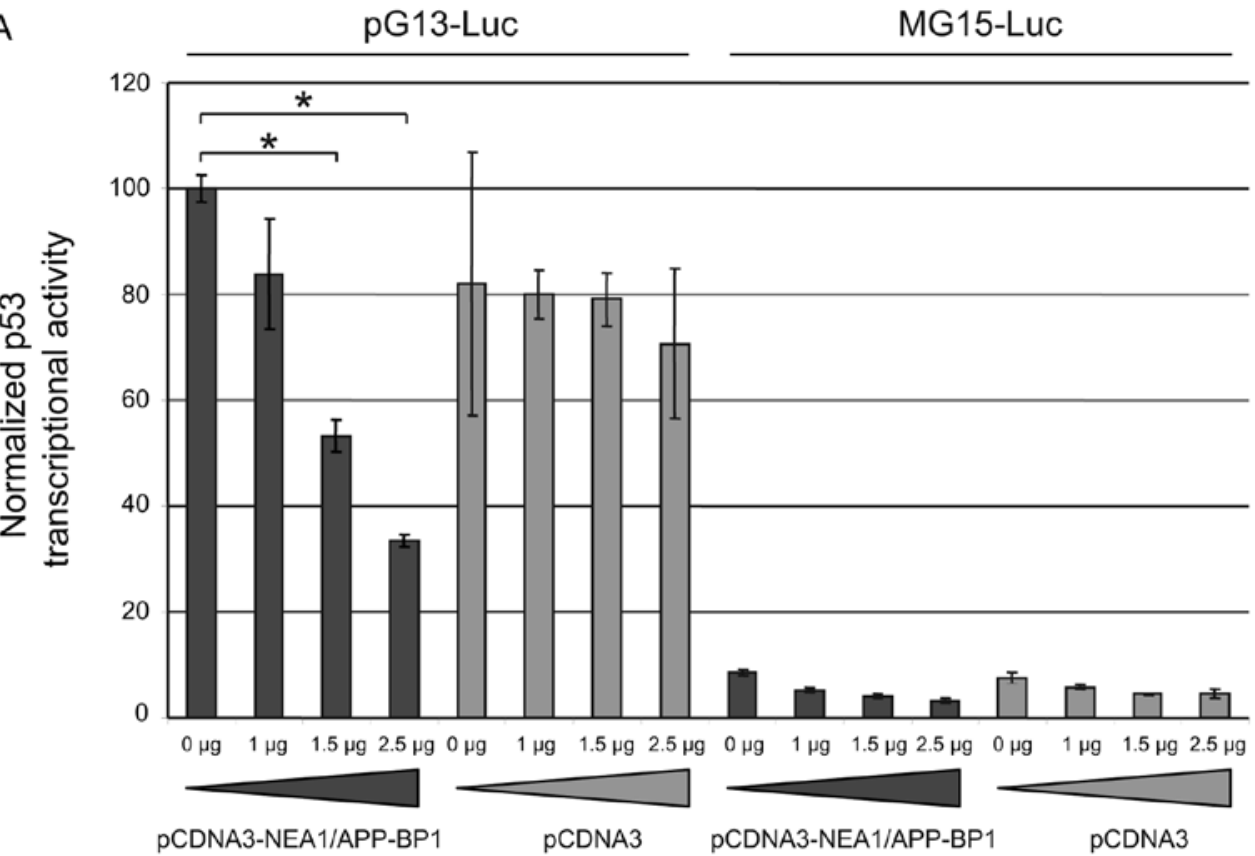

B

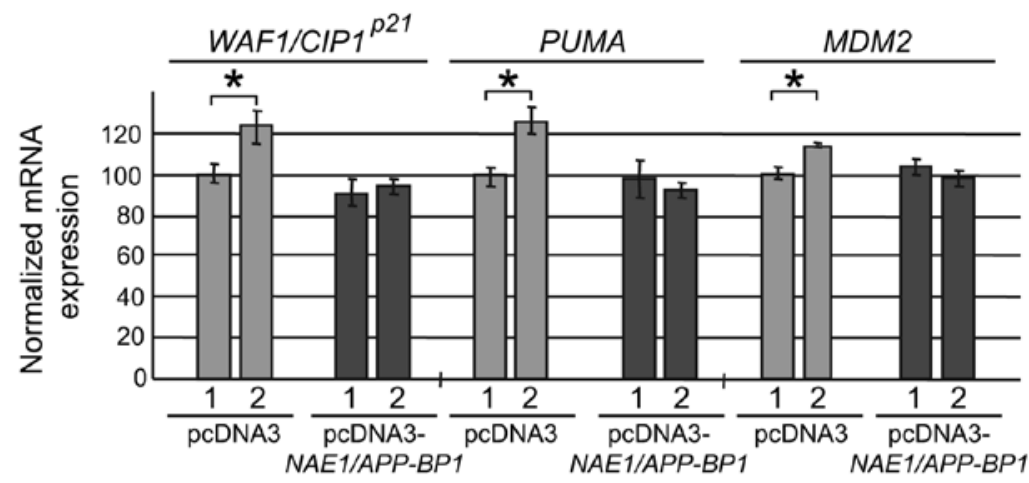

1: non irradiated

2: $30 \mathrm{~min}$

C a

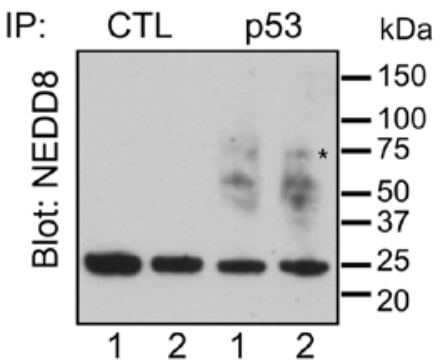

b

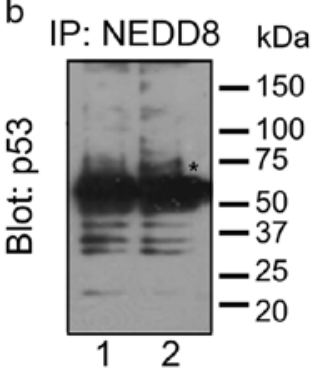

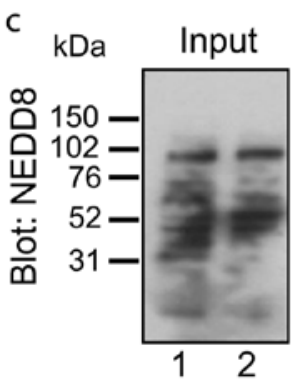

1. UPCI:SCC90 mock transfection 2. UPCI:SCC90 + pCDNA3-NAE1/APP-BP1

*NEDD8-p53

Figure 4. Overexpression of NAE1/APP-BP1 in UPCI:SCC90 cells inhibits p53 transcriptional activity. (A) UPCI:SCC90 cells were transfected with the pG13-Luc reporter vector and increasing amounts $(0,1,1.5$ and $2 \mu \mathrm{g})$ of either the $N A E 1 / A P P-B P 1$ expression construct (dark grey bars) or with the empty expression construct as the negative control (light grey bars). p53 transcriptional activity was measured using luciferase assays in 3 independent samples. Mean p53 transcriptional activity and standard deviations were calculated for each sample. Results were normalized with respect to the non-transfected UPCI:SCC 90 sample, whose value was set to 100 . Similar experiments were performed by using a MG15-Luc reporter gene as the negative control. " $\mathrm{p}<0.05$ indicates statistically significant differences. (B) The expression levels of the WAF1/CIPP ${ }^{21}$, PUMA and MDM2 genes were measured by qRT-PCR in UPCI:SCC90 cells transfected with either the empty pCDNA3 vector (light grey bars) or the NAE1/APP-BP1 expression construct (dark grey bars), in 3 independent experiments. Cells were harvested prior to (1) and $30 \mathrm{~min}$ (2) after a 2 Gy irradiation. Mean gene expression and standard deviations were calculated for each sample. Results were normalized with respect to the mock-transfected UPCI:SCC90 sample, whose value was set to 100 . "p $<0.05$ indicates statistically significant differences. (C) In vivo NEDDylation levels of p53 were evaluated in UPCI:SCC90 cells transfected with either the pCDNA3 empty vector (lane 1) and the NAE1/APP-BP1 expression construct (lane 2). (a) Protein extracts were immunoprecipitated with either a control IgG (left lanes), or an anti-NEDD8 antibody (right lanes). Purified proteins were resolved by SDS-PAGE and membranes were probed with an anti-p53 antibody. "A band corresponding to NEDD8-p53 $(63 \mathrm{kDa})$ is observed. (b) Protein extracts were immunoprecipitated with an anti-p53 antibody and membranes were probed with an anti-NEDD8 antibody. ${ }^{*} \mathrm{~A}$ band corresponding to NEDD8-p53 $(63 \mathrm{kDa})$ is observed. (c) The protein input was evaluated by resolving protein extracts by SDS-PAGE and membranes were probed with an anti-NEDD8 antibody. 
A

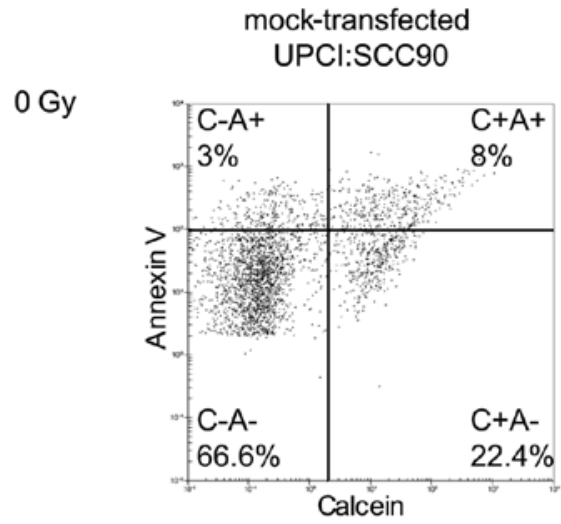

4 Gy

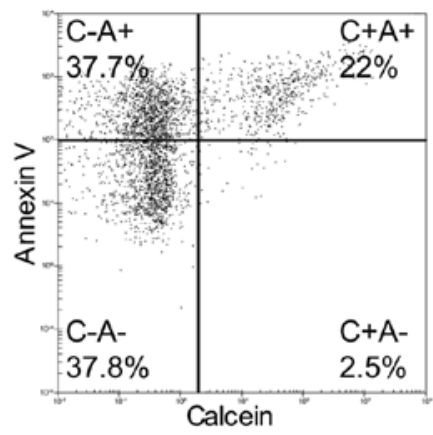

UPCI:SCC90 + pcDN3-NAE1/APP-BP1
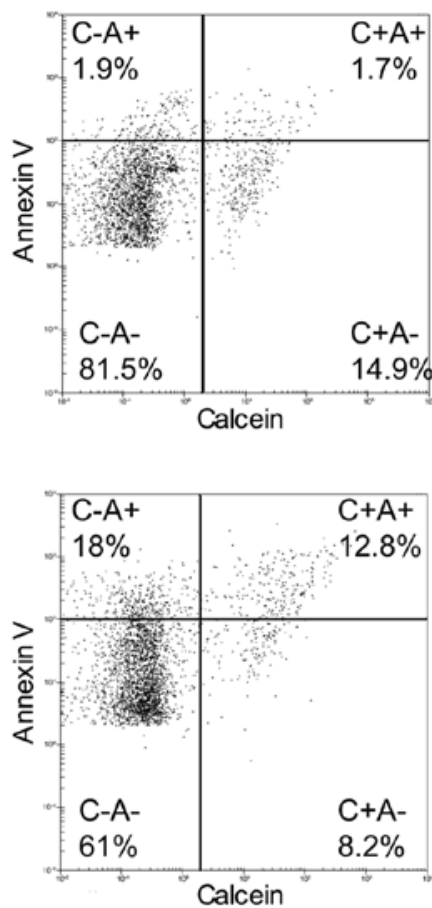

B

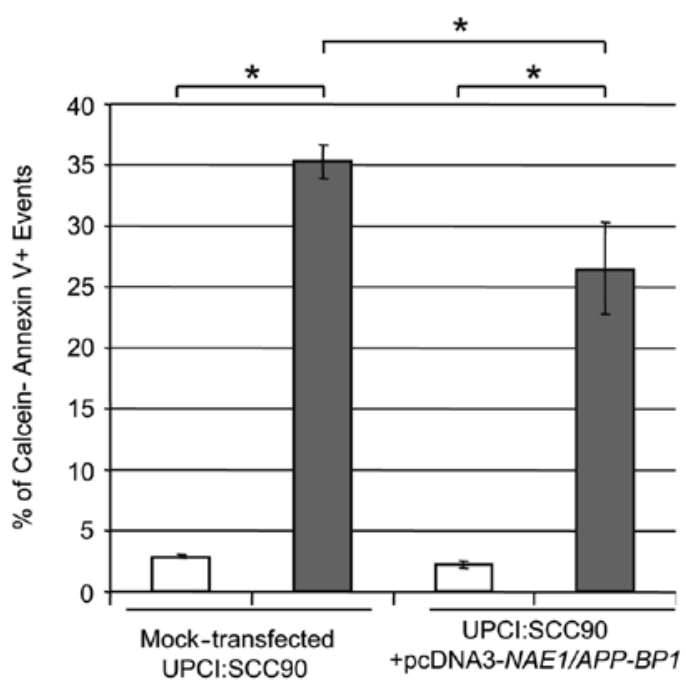

Figure 5. Apoptosis detection by Annexin V staining of transfected and irradiated UPCI:SCC90 cells. (A) Mock-transfected UPCI:SCC90 cells (left panels) and UPCI:SCC90 cells transfected with the NAE1/APP-BP1 expression construct (right panels) were stained with Annexin V and calcein probes prior to (top panels) and $6 \mathrm{~h}$ after a $4 \mathrm{~Gy}$ irradiation (bottom panels). The percentage of each cell categories in one representative experiment is shown (C-A-, calcein-negative Annexin V-negative; C+A-, calcein-positive Annexin V-negative; C-A+, calcein-negative Annexin V-positive; C+A+, calcein-positive Annexin V-positive). (B) Statistical analysis and representation of the calcein-negative Annexin V-positive events observed in mock-transfected cells (left bars) and cells that overexpress NAE1/APP-BP1 (right bars), prior to (white bars) and after irradiation (grey bars). Mean and standard deviation were calculated on 3 independent experiments. P-values were calculated with a Student-Newman-Keuls pairwise comparison. "Differences were considered significant when $\mathrm{p}<0.05$.

and G2 phase distribution of UPCI:SCC90 cells that overexpress NAE1/APP-BP1 (data not shown), suggesting that the effects were not due to changes in the cell cycle.

As a consequence of our findings, the correlation between $N A E / A P P-B P 1$ expression levels and local control and disease-free survival of irradiated patients is currently being investigated. More generally, several studies have unambiguously demonstrated that HR-HPV is a major prognostic factor with respect to both disease-free and overall survival, and this prognosis is independent of treatment modality (1). The question as to whether HPV-positive patients should receive dose de-escalation (decreased irradiation dose delivered to the tumor area, or decreased area that receives the irradiation dose) in order to improve the therapeutic index, while sparing them long-term toxicity, is currently a matter of debate (29). Alternatively, HPV-positive tumors could be managed by modulating existing therapies, in order to potentiate the efficacy of ionizing radiation. 
Our data highlight the relevance of the NEDDylation pathway to the cellular response to radiotherapy, and indicate this pathway is a potential drug target to potentiate existing therapies. Recently, a novel class of pharmacological inhibitors of the NAE1/APP-BP1/UBA3 complex has been tested in preclinical and clinical tumor models $(27,28)$. In the light of our findings, it would be of interest to determine whether a functional copy of $\mathrm{p} 53$ is required for the efficacy of this novel treatment. In addition, these kind of therapeutic approaches could improve the efficacy of the treatment delivered to wild type TP53 HPV-negative tumors.

HR-HPV detection (by immunohistochemistry) has been incorporated into an algorithm that could be used to predict overall survival (29). A stratification of patients based on that type of algorithm would be a first step to randomized trials. In HPV-related oropharyngeal cancer, the loss of genetic material in the $16 \mathrm{q}$ region is related to improved local control $(3,4)$. It would be of great interest to further dissect this locus, in order to identify molecular markers that predict tumor response to therapy.

Besides increasing our knowledge of the molecular mechanisms of cell sensitivity to ionizing radiation, the identification of relevant biomarkers, combined with already established clinical features, may provide physicians with tools to select patients for tailored and more appropriate treatment strategies.

\section{Acknowledgements}

The UPCI:SCC90 cells were kindly provided by Professor Susan Gollin. The pcDNA3-NAEl/APP-BPI vector was a kind gift from Dr Dimitris Xirodimas. The PG13-luc plasmid was a kind gift from Dr Arnold Levine. We thank DrErwan Pencreach for critically reading the manuscript. We are most grateful to Mrs. Christine Wasylyk for her invaluable help with the immunoprecipitation experiments and luciferase assays. S.G. is grateful to the Institut National du Cancer for its support (grant: 'Soutien à la formation à la recherche translationnelle en cancérologie d'étudiants en médecine et de jeunes médecins'). Financial support for this study was provided by the Université de Strasbourg and by the Comité Départemental du Bas-Rhin de la Ligue Contre le Cancer. We are most grateful to the 'Carte d'Identité des Tumeurs' program of the Ligue Nationale contre le Cancer for their long-standing support.

\section{References}

1. Leemans CR, Braakhuis BJ and Brakenhoff RH: The molecular biology of head and neck cancer. Nat Rev Cancer 11: 9-22, 2011.

2. Butz K, Whitaker N, Denk C, Ullmann A, Geisen C and Hoppe-Seyler F: Induction of the p53-target gene GADD45 in HPV-positive cancer cells. Oncogene 18: 2381-2386, 1999.

3. Jung AC, Briolat J, Millon R, et al: Biological and clinical relevance of transcriptionally active human papillomavirus (HPV) infection in oropharynx squamous cell carcinoma. Int J Cancer 126: 1882-1894, 2010.

4. Klussmann JP, Mooren JJ, Lehnen M, et al: Genetic signatures of HPV-related and unrelated oropharyngeal carcinoma and their prognostic implications. Clin Cancer Res 15: 1779-1786, 2009.

5. Gong $\mathrm{L}$ and Yeh ET: Identification of the activating and conjugating enzymes of the NEDD8 conjugation pathway. J Biol Chem 274: 12036-12042, 1999.
6. Osaka F, Kawasaki H, Aida N, et al: A new NEDD8-ligating system for cullin-4A. Genes Dev 12: 2263-2268, 1998.

7. Petroski MD and Deshaies RJ: Function and regulation of cullinRING ubiquitin ligases. Nat Rev Mol Cell Biol 6: 9-20, 2005.

8. Xirodimas DP, Saville MK, Bourdon JC, Hay RT and Lane DP: Mdm2-mediated NEDD8 conjugation of p53 inhibits its transcriptional activity. Cell 118: 83-97, 2004.

9. Ragin CC, Reshmi SC and Gollin SM: Mapping and analysis of HPV16 integration sites in a head and neck cancer cell line. Int J Cancer 110: 701-709, 2004.

10. Maalouf M, Alphonse G, Colliaux A, et al: Different mechanisms of cell death in radiosensitive and radioresistant p53 mutated head and neck squamous cell carcinoma cell lines exposed to carbon ions and x-rays. Int J Radiat Oncol Biol Phys 74: 200-209, 2009

11. Gupta AK, Lee JH, Wilke WW, et al: Radiation response in two HPV-infected head-and-neck cancer cell lines in comparison to a non-HPV-infected cell line and relationship to signaling through AKT. Int J Radiat Oncol Biol Phys 74: 928-933, 2009.

12. Chen Y, McPhie DL, Hirschberg J and Neve RL: The amyloid precursor protein-binding protein APP-BP1 drives the cell cycle through the S-M checkpoint and causes apoptosis in neurons. J Biol Chem 275: 8929-8935, 2000.

13. de Boer MA, Jordanova ES, Kenter GG, et al: High human papillomavirus oncogene mRNA expression and not viral DNA load is associated with poor prognosis in cervical cancer patients. Clin Cancer Res 13: 132-138, 2007.

14. Garrison SP, Jeffers JR, Yang C, et al: Selection against PUMA gene expression in Myc-driven B-cell lymphomagenesis. Mol Cell Biol 28: 5391-5402, 2008.

15. Franken NA, Rodermond HM, Stap J, Haveman J and van Bree C: Clonogenic assay of cells in vitro. Nat Protoc 1: 2315-2319, 2006.

16. Abramoff MD, Magelhaes PJ and Ram SJ: Image processing with ImageJ. Biophotonics Int 11: 36-42, 2004.

17. Rasband WS: ImageJ. National Institutes of Health, Bethesda, MA. http://rsb.info.nih.gov/ij/, 1997-2008.

18. Kozarsky KF, McKinley DR, Austin LL, Raper SE, StratfordPerricaudet LD and Wilson JM: In vivo correction of low density lipoprotein receptor deficiency in the Watanabe heritable hyperlipidemic rabbit with recombinant adenoviruses. J Biol Chem 269: 13695-13702, 1994.

19. Lassen P: The role of Human papillomavirus in head and neck cancer and the impact on radiotherapy outcome. Radiother Oncol 95: 371-380, 2010.

20. Abida WM, Nikolaev A, Zhao W, Zhang W and Gu W: FBXO11 promotes the Neddylation of p53 and inhibits its transcriptional activity. J Biol Chem 282: 1797-1804, 2007.

21. Kim HJ, Kim SH, Shim SO, et al: Drosophila homolog of APP-BP1 (dAPP-BP1) interacts antagonistically with APPL during Drosophila development. Cell Death Differ 14: 103-115, 2007.

22. Nakayama KI and Nakayama K: Ubiquitin ligases: cell-cycle control and cancer. Nat Rev Cancer 6: 369-381, 2006.

23. Jia L, Yang J, Hao X, et al: Validation of SAG/RBX2/ROC2 E3 ubiquitin ligase as an anticancer and radiosensitizing target. Clin Cancer Res 16: 814-824, 2010.

24. Watson IR, Blanch A, Lin DC, Ohh M and Irwin MS: Mdm2mediated NEDD8 modification of TAp73 regulates its transactivation function. J Biol Chem 281: 34096-34103, 2006.

25. Quiet CA, Weichselbaum RR and Grdina DJ: Variation in radiation sensitivity during the cell cycle of two human squamous cell carcinomas. Int J Radiat Oncol Biol Phys 20: 733-738, 1991.

26. Tell R, Heiden T, Granath F, Borg AL, Skog S and Lewensohn R: Comparison between radiation-induced cell cycle delay in lymphocytes and radiotherapy response in head and neck cancer. Br J Cancer 77: 643-649, 1998.

27. Milhollen MA, Traore T, Adams-Duffy J, et al: MLN4924, a NEDD8-activating enzyme inhibitor, is active in diffuse large B-cell lymphoma models: rationale for treatment of NF- $\kappa \mathrm{B}-$ dependent lymphoma. Blood 116: 1515-1523, 2010.

28. Soucy TA, Smith PG, Milhollen MA, et al: An inhibitor of NEDD8-activating enzyme as a new approach to treat cancer. Nature 458: 732-736, 2009.

29. Ang KK, Harris J, Wheeler R, et al: Human papillomavirus and survival of patients with oropharyngeal cancer. N Engl J Med 363: 24-35, 2010. 